\title{
Effective color transfer enables rapid computational microscopy for digital pathology
}

\author{
ShaoWei Jiang, and GuoAn Zheng* \\ Department of Biomedical Engineering, University of Connecticut, Storrs 06269, USA
}

Received August 30, 2021; accepted September 2, 2021; published online September 28, 2021

Citation: $\quad$ S. W. Jiang, and G. A. Zheng, Effective color transfer enables rapid computational microscopy for digital pathology, Sci. China-Phys. Mech. Astron.
$\quad 64,114231$ (2021), https://doi.org/10.1007/s11433-021-1782-3

In most pathology labs, clinicians diagnose diseases by examining tissue slices using a light microscope. This process typically requires clinicians to move the microscope stage to different positions and identify areas of interest that can be further analyzed using a higher magnification objective lens. For proper focusing of the slide, the axial position needs to be constantly adjusted by manually rotating the focus knob. As a result, the reviewing process can be easily disrupted when the clinician bumps the slide to the objective lens or switches to a different objective lens for focusing again. Although it remains the gold standard in diagnosing almost all types of cancers, manual microscopic inspection is, in general, labor-intensive and does not form a streamlined workflow in clinical practice. Furthermore, it is largely based on subjective opinions of clinicians: different clinicians may arrive at different conclusions for the same slide and the same person may give different conclusions at different time points [1].

One solution to bring the manual inspection process into digital practice is image acquisition of the entire tissue section for virtual inspection. Clinicians can then navigate, change magnification, and annotate the virtual slide with speed and ease. Whole slide imaging based on robotic microscopy is now advancing into the clinical workflow for better and faster predication and diagnosis of cancers and other diseases [1]. A regular whole slide imaging system

*Corresponding author (email: guoan.zheng@uconn.edu) acquires many high-resolution images and stitches them in the spatial domain, like the panorama mode of a cellphone camera. The system, however, requires precise mechanical control, is expensive and of high maintenance, and is not compatible with most existing microscopes in laboratories. It also lacks the flexibility for imaging biospecimens other than regular microscope slides.

The latest digital tools of computational microscopy make it possible to acquire high-resolution, wide-field digital equivalent of the original glass slide for virtual inspection [24]. Fourier ptychographic microscopy (FPM) is one example that converts a regular microscope into a gigapixel imaging tool without involving mechanical scanning [2,5]. It can be built using a regular microscope platform with a low magnification objective lens and a programmable LED array [2,5-8]. By turning on individual LED elements on the array, the system illuminates the sample from different incident angles with partially coherent light. The image recorded at each angle corresponds to the information from a circular pupil aperture in the Fourier domain. The size of the aperture is determined by the numerical aperture of the objective while its offset from the origin is determined by the illumination wave vector. In contrast with the regular whole slide imaging system, FPM stitches all captured images in the Fourier domain using an iterative phase retrieval process. The final recovery, therefore, has a substantially expanded passband for resolution improvement and retains the original large field of view set by the low magnification objective lens. 

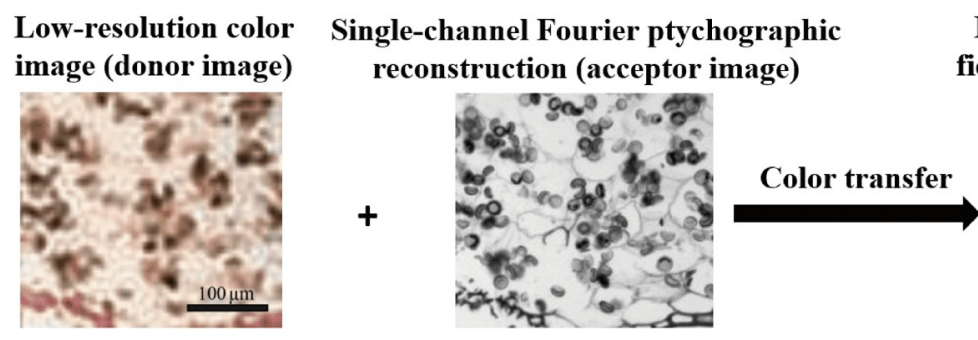

High-resolution, large

field-of-view color image

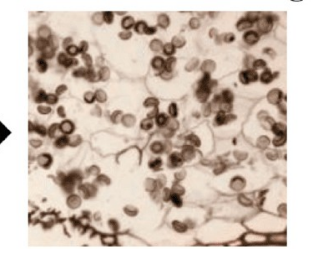

Figure 1 (Color online) Effective color transfer for rapid FPM imaging. Two inputs images are acquired using an FPM platform: (1) a low-resolution color image via red, green, and blue LED illuminations, and (2) a high-resolution FPM reconstruction at the green wavelength. These two images are used to create a high-resolution, large field-of-view color image of sample. Since only one high-resolution channel is needed in this strategy, it can substantially reduce the acquisition and reconstruction time for FPM. Modified from ref. [9].

One obstacle for adopting FPM and other coherent imaging modalities in digital pathology is the acquisition of color information. The original FPM approach accomplishes this by sequentially recovering object images under red, green, and blue LED illuminations [2]. This strategy is not time efficient due to the repeated acquisitions at different wavelengths. The coherence nature of this computational microscopy technique also leads to color artifacts caused by dust particles on the slide and lenses. The background of the recovered images is, in general, not as clean as that captured with incoherent light.

To address the challenge of color information recovery in coherent microscopy, Gao et al. [9] reported an effective color transfer approach for rapid FPM imaging. This approach is developed based on the concept of color matching, where the hue of the donor image is applied to the acceptor image. Figure 1 shows the schematic of this approach, where two images are acquired using an FPM setup. The donor image is the low-resolution color image captured by sequentially turning on the red, green, and blue LEDs for sample illumination. The acceptor image is the high-resolution FPM reconstruction at the green wavelength. By performing color transfer, these two inputs images can generate the high-resolution color image on the right panel of Figure 1.

In the implementation by Gao et al. [9], they first convert the sRGB color space into the $L a b$ color space, where $L$ is the channel representing brightness. The color texture information of the donor image is then transferred to the acceptor image of the single-channel FPM reconstruction. Lastly, the $L a b$ color model of the FPM reconstruction is converted back to the sRGB space for display.

The accuracy and efficiency of this color transfer scheme are validated by imaging 30 different biospecimens. The error metric shows that the color transfer method has a similar performance as that of the conventional sequential acquisition method. More interestingly, the color transfer method outperforms the sequential acquisition method when the sample image contains a high background. The reason is that the FPM system has chromatic aberrations when acquired at different wavelengths. The color-transfer approach is less affected because the acceptor image is recovered at a single wavelength.

One drawback of this color transfer approach, perhaps, is that it has not utilized the spatially matched information between the donor image and the acceptor image. The donor image is only used for extracting the color texture, and the staining process is not supervised by the spatial information of the donor. As a result, the current implementation cannot distinguish multiple dyes. For example, the red stains in Figure 1 cannot be properly transferred to the output image. To improve the color imaging performance, we envision a better staining scheme that transfers the color texture and enforces the paired data constraint between the donor and acceptor. Such a scheme will greatly facilitate the adoption of computational microscopy in digital pathology applications.

1 F. Ghaznavi, A. Evans, A. Madabhushi, and M. Feldman, Annu. Rev. Pathol. Mech. Dis. 8, 331 (2013).

2 G. Zheng, R. Horstmeyer, and C. Yang, Nat. Photon. 7, 739 (2013), arXiv: 1405.0226.

3 A. Greenbaum, Y. Zhang, A. Feizi, P. L. Chung, W. Luo, S. R. Kandukuri, and A. Ozcan, Sci. Trans. Med. 6, 267 ra175 (2014).

4 P. Song, R. Wang, J. Zhu, T. Wang, Z. Bian, Z. Zhang, K. Hoshino, M. Murphy, S. Jiang, C. Guo, and G. Zheng, Opt. Lett. 45, 3486 (2020), arXiv: 2006.08114.

5 G. Zheng, C. Shen, S. Jiang, P. Song, and C. Yang, Nat. Rev. Phys. 3, 207 (2021)

6 X. Ou, G. Zheng, and C. Yang, Opt. Express 22, 4960 (2014).

7 L. Tian, X. Li, K. Ramchandran, and L. Waller, Biomed. Opt. Express 5, 2376 (2014).

8 A. Pan, Y. Zhang, K. Wen, M. Zhou, J. Min, M. Lei, and B. Yao, Opt. Express 26, 23119 (2018), arXiv: 1806.05636.

9 Y. Gao, J. Chen, A. Wang, A. Pan, C. Ma, and B. Yao, Sci. ChinaPhys. Mech. Astron. 64, 114211 (2021). 